\title{
A Bayesian Approach to Competing Risks Model with Masked Causes of Failure and Incomplete Failure Times
}

\author{
Yosra Yousif, ${ }^{1}$ Faiz A. M. Elfaki $\mathbb{D}^{\circ},{ }^{2}$ Meftah Hrairi, ${ }^{1}$ and Oyelola A. Adegboye $\mathbb{D}^{3,4}$ \\ ${ }^{1}$ Department of Mechanical Engineering, Faculty of Engineering, International Islamic University Malaysia, \\ Kuala Lumpur, Malaysia \\ ${ }^{2}$ Department of Mathematics, Statistics and Physics, College of Arts and Sciences, Qatar University, Doha, Qatar \\ ${ }^{3}$ Evolution Equations Research Group, Ton Duc Thang University, Ho Chi Minh City, Vietnam \\ ${ }^{4}$ Faculty of Mathematics and Statistics, Ton Duc Thang University, Ho Chi Minh City, Vietnam \\ Correspondence should be addressed to Faiz A. M. Elfaki; felfaki@qu.edu.qa
}

Received 26 June 2019; Revised 26 January 2020; Accepted 13 February 2020; Published 24 April 2020

Academic Editor: Paolo Spagnolo

Copyright $(2020$ Yosra Yousif et al. This is an open access article distributed under the Creative Commons Attribution License, which permits unrestricted use, distribution, and reproduction in any medium, provided the original work is properly cited.

\begin{abstract}
We present a Bayesian approach for analysis of competing risks survival data with masked causes of failure. This approach is often used to assess the impact of covariates on the hazard functions when the failure time is exactly observed for some subjects but only known to lie in an interval of time for the remaining subjects. Such data, known as partly interval-censored data, usually result from periodic inspection in production engineering. In this study, Dirichlet and Gamma processes are assumed as priors for masking probabilities and baseline hazards. Markov chain Monte Carlo (MCMC) technique is employed for the implementation of the Bayesian approach. The effectiveness of the proposed approach is illustrated with simulated and production engineering applications.
\end{abstract}

\section{Introduction}

In survival analysis, competing risks models are often used when multiple potential causes of failure are observed. Often, the causes of failure are unidentified $[1,2]$. Consider the example shown in Reiser et al. [1]. Out of the 682 IBM PS/2 computer system units tested (a system is made of three components: motherboard, disc drives, and power supply), eight failed and the remaining 674 were right censored at various times [1]. The eight systems failed due to malfunction in at least one of the three components $\{1,2,3\}$ and often the cause is narrowed down to several possibilities, e.g., $\{1,3\}$, indicating that the cause of failure is either the motherboard or power supply [1]. This type of incomplete data is generally referred to as masked data, where the cause of failure can only be identified up to a minimum random subset (MRS) $S \subseteq\{1, \ldots, K\}$.

Let $T$ be the time until a unit experiences a failure due to one of the $K$ possible causes of failure. If the precise cause of failure is identified as $K$, then $S=\{K\}$ is a singleton. If the cause of failure is not identified, then $S=\{1, \ldots, K\}$, resulting in full masking of the cause. Thus, every observed failure time $T_{i}, i=1, \ldots, N$, is accompanied by a observed MRS denoted by $S_{i}$. However, another reason for incomplete observations could be due to the unavailability of knowledge about the exact failure time $T$.

There is abundant literature of masked data [1-9]. Miyakawa [8] provided maximum likelihood estimates (MLEs) with two causes of failure and independent exponential failure times when the data is masked and uncensored. Dinse [9] suggested nonparametric maximum likelihood estimators of prevalence and mortality when the MRS is a known cause or a full masked cause.

In production engineering, researchers were often interested in the assessment of components reliability. When the impact of the risk factors on the hazard function is of interest, Bayesian analysis under cause-specific hazard framework is often considered; see, for example, [1-7]. Bayesian approach is preferred as it is flexible in allowing a general pattern of masking as well as relaxing assumptions 
[2]. In their work, Reiser et al. [1] presented a Bayesian analysis assuming exponentially distributed component lifetimes, and Guttman et al. [7] took this work further to the case where the masking probability depends on the actual cause of failure. Considering the partial masking cases, Mukhopadhyay and Basu [4] explored a Bayesian approach with independent Weibull distributions and made the assumption of identical shape structures for all the $K$ risks. On the other hand, Basu et al. [6] used a Bayesian analysis for masked data from a general $K$ component system with nonidentical Weibull distributions. In their follow-up study, Basu et al. [5] explored a Bayesian approach based on a general flexible parametric framework for complex forms of censoring. Without the symmetry assumption, Kuo and Yang [10] developed a Bayesian analysis with independent exponential as well as Weibull distributions, while Mukhopadhyay and Basu [3] studied the case of a series system, the components of which follow independent lognormal life distribution. Xu and Tang [11] considered a nonparametric Bayesian approach for masked data which extended the findings of Neath and Samaniego [12] to series systems with partially masked competing risks. In contrast and based on a cause-specific formulation, Flehinger et al. [13] proposed an approach of completely parametric causespecific hazards using stage 1 and stage 2 information when the failure times for the competing risks have a Weibull distribution. In their work, Craiu and Reiser [14] developed an expectation-maximization- (EM-) based method that allowed dependent competing risks and produced estimators for the subdistribution functions. Similarly, $\mathrm{Lu}$ and Tsiatis [15] presented parametric models to estimate the regression coefficients whereby the cause-specific hazard for the cause of interest is associated with the covariates through a proportional hazards relationship. Sen et al. [2] introduced a semiparametric Bayesian approach using variety in priors.

Most of the works mentioned above were developed for masked data based on the series of system formulation considering cases where the failure time was complete (no censored units), right-censored (RC), or interval-censored. In this paper, we investigated the case where the data is masked and the failure time is partly interval-censored (PIC) using the Bayesian approach. We employed the Cox proportional hazards model, which is used extensively but mostly for public health studies; see, for example, Han et al. [16] and Liu et al. [17].

The rest of the paper is organized as follows. Section 2 introduces the model formulation and Bayesian computation techniques. Section 3.1 provides some results from modest simulation to evaluate the model performance. The illustration of our approach using an actual data set were presented in Section 3.2 Section 4 concludes the paper.

\section{Materials and Methods}

2.1. Model Structure. In masked data, we do not only observe the failure time for every unit but also a set of causes that contains the true cause of failure. Assume that we observe $N$ units each with $K$ causes of failure acting on it. Let $X$ denote the observed collection of covariates, then for any unit $i$, we observe the vector $\left(T_{i}, S_{i}, X_{i}\right)$, where $T_{i}$ denotes the failure time and $S_{i}$ denotes the minimum random subset (MRS) of causes that are possibly responsible for the unit failure. Following Kuo and Yang [10], for the $i^{\text {th }}$ unit, the likelihood contribution from the data $\left(T_{i}, S_{i}, X_{i}\right)$ consists in $P\left(T_{i}, S_{i} \mid X_{i}\right)$ can be expressed as

$$
P\left(T_{i}, S_{i} \mid X_{i}\right)=P\left(T_{i}, C_{i}=j \mid X_{i}\right) P\left(S_{i} \mid T_{i}, C_{i}=j, X_{i}\right), \quad j=1, \ldots, K,
$$

where $C_{i}$ denotes the actual cause of failure of the $i^{\text {th }}$ unit. Note that $P\left(T_{i}, C_{i}=j \mid X_{i}\right)=f_{j}\left(T_{i} \mid X_{i}\right)$. When the observation of $C$ is incomplete (see Crowder [12]), the likelihood contribution for an observed failure can be modified to

$$
\sum_{j \in S i} P\left(T_{i}, S_{i} \mid X_{i}\right)=\sum_{j \in S i} f_{j}\left(T_{i} \mid X_{i}\right) P\left(S_{i} \mid T_{i}, C_{i}=j, X_{i}\right) .
$$

In the case of partly interval-censored data where the time of failure is incomplete, but observe the exact failure time for some units, however only the interval of time that includes the true failure time for the remaining units is observed. This type of data often arises in production engineering when units are inspected periodically. Let $\left(L_{i}, R_{i}\right]$, where $L_{i}<R_{i}$, denotes the observed interval including the true failure time of the $i^{\text {th }}$ unit $\left(T_{i} \in\left(L_{i}, R_{i}\right]\right)$. If the unit failure occurs before the first inspection time, then we have a left-censored observation $\left(T_{i} \in\left(0, R_{i}\right]\right)$; if the unit does not fail until the last inspection time, then we have a rightcensored observation $\left.\left(T_{i} \in L_{i}, \infty\right]\right)$. Define $\delta_{i}, \gamma_{i}$ as censoring indicators taking the value of one if the failure time $T_{i}$ is left-censored or interval-censored and taking a value of zero otherwise. Then, the likelihood contribution of the $i^{\text {th }}$ unit when the observation of $T$ is incomplete can be expressed as

$$
L=\prod_{i=1}^{n} f\left(T_{i} \mid X_{i}\right) \prod_{i=n+1}^{m}\left[S\left(L_{i} \mid X_{i}\right)-S\left(R_{i} \mid X_{i}\right)\right], L=\prod_{i=1}^{n} f\left(T_{i} \mid X_{i}\right) \prod_{i=n+1}^{m}\left[1-S\left(R_{i} \mid X_{i}\right)\right]^{\delta_{i}}\left[\left(\frac{S\left(R_{i} \mid X_{i}\right)}{S\left(L_{i} \mid X_{i}\right)}\right)-1\right]^{\gamma_{i}}\left[S\left(L_{i} \mid X_{i}\right)\right]^{1-\delta_{i}} .
$$

where $n$ and $m(n+m=N)$ are the numbers of the units whose failure time is exact and interval-censored (including left- and right-censored), respectively.
In this study, we used semiparametric Bayesian approach to estimate the regression coefficients for the set of covariates. Further, we adopted the cause-specific formulation 
utilizing the popular proportional hazards $(\mathrm{PH})$ model that is of the form

$$
\lambda_{j}(T, X)=\lambda_{0 j}(T) e^{\beta_{j}^{\prime} X}, \quad j=1, \ldots, K,
$$

where $\lambda_{0 j}$ and $\beta_{j}$ are the baseline hazard and the regression coefficient of the $i^{\text {th }}$ cause of failure and $X$ represents the vector of the covariates, respectively.
In this study, both failure time and cause of failure are incomplete; therefore, we need to consider the two cases discussed above to formulate the likelihood function. Let $n_{1}, n_{2}, n_{3} \quad\left(n_{1}+n_{2}+n_{3}=N\right)$ denote the numbers of the units whose failure times are exact, right-censored, and interval-censored (including left-censored), respectively. Then, the full likelihood of masked and partly intervalcensored data can be expressed as

$$
\begin{aligned}
L= & \prod_{i=1}^{n_{1}} \sum_{j \in S_{i}} P\left(S_{i} \mid T_{i}, C_{i}=j, X_{i}\right) f_{j}\left(T_{i} \mid X_{i}\right) \prod_{i=n_{1}+1}^{n_{2}} S\left(L_{i} \mid X_{i}\right) \times \prod_{i=n_{2}+1}^{n_{3}} \sum_{j \in S_{i}} P\left(S_{i} \mid T_{i}, C_{i}=j, X_{i}\right)\left[F_{j}\left(R_{i} \mid X_{i}\right)-F_{j}\left(L_{i} \mid X_{i}\right)\right], \\
L= & \prod_{i=1}^{n_{1}} \sum_{j \in S_{i}} P\left(S_{i} \mid T_{i}, C_{i}=j, X_{i}\right) \lambda_{j}\left(T_{i} \mid X_{i}\right) e^{-\sum_{j=1}^{K} \int_{0}^{T_{i}} \lambda_{j}\left(t \mid X_{i}\right) \mathrm{d} t} \times \prod_{i=n_{1}+1}^{n_{2}} e^{-\sum_{j=1}^{K} \int_{0}^{T_{i}} \lambda_{j}\left(t \mid X_{i}\right) \mathrm{d} t} \\
& \times \prod_{i=n_{2}+1}^{n_{3}} \sum_{j \in S_{i}} P\left(S_{i} \mid T_{i}, C_{i}=j, X_{i}\right)\left[\int_{0}^{R_{i}} \lambda_{j}\left(t \mid X_{i}\right) e^{-\sum_{j=1}^{K} \int_{0}^{T_{i}} \lambda_{j}\left(s \mid X_{i}\right) \mathrm{d} t} \mathrm{~d} t-\int_{0}^{L_{i}} \lambda_{j}\left(t \mid X_{i}\right) e^{-\sum_{j=1}^{K} \int_{0}^{T_{i}} \lambda_{j}\left(s \mid X_{i}\right) \mathrm{d} s} \mathrm{~d} t\right] .
\end{aligned}
$$

Substituting equation (4) in (5), the full-likelihood function emerges as

$$
\begin{aligned}
L= & \prod_{i=1}^{n_{1}} \sum_{j \in S_{i}} P\left(S_{i} \mid T_{i}, C_{i}=j, X_{i}\right) \lambda_{0 j}\left(T_{i}\right) e^{\beta_{j}^{\prime} X} e^{-\sum_{j=1}^{K} \int_{0}^{T_{i}} \lambda_{0 j}(t) e^{\beta_{j}^{\prime} X}} \mathrm{~d} t \times \prod_{i=n_{1}+1}^{n_{2}} e^{-\sum_{j=1}^{K} \int_{0}^{T_{i}} \lambda_{j}\left(t \mid X_{i}\right) \mathrm{d} t} \\
& \times \prod_{i=n_{2}+1}^{n_{3}} \sum_{j \in S_{i}} P\left(S_{i} \mid T_{i}, C_{i}=j, X_{i}\right)\left[\int_{0}^{R_{i}} \lambda_{0 j}(t) e^{\beta_{j}^{\prime} X} e^{-\sum_{j=1}^{K} \int_{0}^{t} \lambda_{0 j}(s) e^{\beta_{j}^{\prime \prime} X}} \mathrm{~d} s \mathrm{~d} t-\int_{0}^{L_{i}} \lambda_{0 j}(t) e^{\beta_{j}^{\prime} X} e^{-\sum_{j=1}^{K} \int_{0}^{t} \lambda_{0 j}(s) e^{\beta_{j}^{\prime} X}} \mathrm{~d} s \mathrm{~d} t\right]
\end{aligned}
$$

When dealing with the masked data, the unrealistic symmetry assumption that involves an equal chance of observing a similarly masked subset of causes regardless of the actual cause is often adopted, i.e.,

$$
P\left(S_{i} \mid T_{i}, C_{i}=j, X_{i}\right)=P\left(S_{i} \mid T_{i}, C_{i}=j^{\prime}, X_{i}\right), \quad j, j^{\prime} \in S_{i} .
$$

Assumption (7) makes the analysis proceed with a reduced likelihood function that is not reliant on the masking probabilities. In contrast, in this paper, we applied a Bayesian analysis, which does not rely on the symmetry assumption [1], using the full likelihood function of equation (6). We model the masking probabilities to be independent of the failure time and dependent on the cause of failure. Moreover, we allow them to depend on subject-level covariates.
It is often of interest to determine the cause that is responsible for the unit failure when it is masked. For this purpose, we need to compute the diagnostic probability, which is the probability of the $i^{\text {th }}$ risk, causing the unit to fail given the observed masking set and the unit's failure time. According to our full likelihood, we have two different ways to compute the diagnostic probabilities depending on whether the failure time of the unit is exact or intervalcensored (including left-censored). First, when the $i^{\text {th }}$ unit is exact, the diagnostic probability can be defined as

$$
P\left(C_{i}=j \mid S_{i}, T_{i}, X_{i}\right)=\frac{P\left(S_{i} \mid T_{i}, C_{i}=j, X_{i}\right) f_{j}\left(T_{i} \mid X_{i}\right)}{\sum_{l \in S_{i}} P\left(S_{i} \mid T_{i}, C_{i}=l, X_{i}\right) f_{l}\left(T_{i} \mid X_{i}\right)}, \quad j \in S_{i} .
$$

Second, when the $i^{\text {th }}$ unit is interval-censored, the diagnostic probability can be defined as

$$
P\left(C_{i}=j \mid S_{i}, T_{i}, X_{i}\right)=\frac{P\left(S_{i} \mid T_{i}, C_{i}=j, X_{i}\right)\left[F_{j}\left(R_{i} \mid X_{i}\right)-F_{j}\left(L_{i} \mid X_{i}\right)\right]}{\sum_{l \in S_{i}} P\left(S_{i} \mid T_{i}, C_{i}=l, X_{i}\right)\left[F_{l}\left(R_{i} \mid X_{i}\right)-F_{l}\left(L_{i} \mid X_{i}\right)\right]}, \quad j \in S_{i} .
$$


TABLE 1: The posterior summaries of the regression coefficients from the two approaches.

\begin{tabular}{lccccccrr}
\hline \multirow{2}{*}{ Parameters } & \multicolumn{3}{c}{ Right-censored (RC) data } & \multicolumn{3}{c}{ Partly interval-censored (PIC) data } \\
& Mean & Median & SE & $95 \%$ PCI $^{*}$ & Mean & Median & SE & 95\% PCI* \\
\hline$\beta_{1}$ & 1.009 & 1.002 & 0.486 & $0.066-1.973$ & 0.774 & 0.768 & 0.473 & $-0.144-1.730$ \\
$\mathrm{~B}_{2}$ & 0.733 & 0.719 & 0.667 & $-0.057-2.072$ & 0.508 & 0.500 & 0.644 & $-0.741-1.803$ \\
\hline
\end{tabular}

${ }^{*}$ Posterior credible interval.

2.2. Bayesian Framework. For semiparametric approaches to Bayesian inference involving hazard regression models, Cox's $\mathrm{PH}$ model is one of the most commonly adopted models. Suppose there are $N$ units under observation with $K$ competing risks acting on them, and the observed data $D=\left\{N_{i j}(t), Y_{i j}(t), X\right\}, i=1, \ldots, N, j=1, \ldots, K$, where $N_{i j}(t)$ is the process counting the failures due to cause $j$ occurring up to time $t$ and $Y_{i j}(t)$ is the at risk indicator for cause $j$. Let $d N_{i j}(t)$ be a small increment of $N_{i j}(t)$ over the interval [ $t$, $t+\mathrm{d} t)$, then $N_{i j}(t)$ and $d N_{i j}(t)$ equal 1 if the event occurs in [0, $t)$ and $[t, t+\mathrm{d} t)$, respectively, and 0 otherwise. Under noninformative censoring, the likelihood (specific to the $j^{\text {th }}$ cause of failure) of the data is proportional to

$$
\prod_{i=1}^{N}\left[\prod_{t \geq 0} I_{i j}(t)^{d N_{i j}(t)}\right] e^{-I_{i j}(t) \mathrm{d} t}
$$

This is basically as if the counting process increments $d N_{i j}(t)$ over the time interval $[t, t+\mathrm{d} t)$ are independent Poisson random variables with means $I_{i j}(t) \mathrm{d} t$. That is,

$$
\begin{gathered}
d N_{i j}(t) \sim \operatorname{Poisson}\left(I_{i j}(t) \mathrm{d} t\right), \\
I_{i j}(t) \mathrm{d} t=Y_{i}(t) e^{\beta_{j}^{\prime} X} d \Lambda_{0 j}(t),
\end{gathered}
$$

where $d \Lambda_{0 j}(t)=\lambda_{0 j}(t) \mathrm{d} t$ is the immediate probability that the unit at risk at time $t$ has the event $j$ in the next time interval $[t, t+\mathrm{d} t)$. After specifying the likelihood form, the interest turns to the prior distributions. We need to define prior distributions to the unknown parameters which are assumed to be stochastically independent. The prior specifications follow standard practice in semiparametric Bayesian analysis where popular priors that conjugate for the likelihood function are utilized. Following Sen et al. [2] and $\mathrm{Xu}$ and Tang [11], we assigned independent Dirichlet priors to the masking probabilities. Let $J=2^{j-1}$ denote the number of sets that include the cause $j$ and let $S_{j}=\left\{S_{j 1}, \ldots, S_{j 2}\right\}$ denote the collection of potential MRS's that contain cause $j$, then the random Dirichlet variables can be defined as

$$
\left(\mu_{i j}\left(S_{j 1}\right), \ldots, \mu_{i j}\left(S_{j J}\right)\right) \sim \operatorname{Dir}_{J}\left(\alpha_{j}\right), \quad i=1, \ldots, N ; j=1, \ldots, K ; J=2^{j-1},
$$

where $\mu_{i j}=P\left(S_{i} \mid T_{i}, C_{i}=j, X_{i}\right)$ and $\alpha_{j}=\left(\alpha_{j 1}, \ldots, \alpha_{j J}\right)$ are the Dirichlet parameters.

Using an independent gamma process for the prior for cause-specific baseline hazards, $\Lambda_{0 j}(t) \backsim \mathrm{GP}\left(c \omega_{j}(t), c\right)$, $j=1, \ldots, K$, where $\Lambda_{0 j}$ is the cumulative baseline hazard specific to $i^{\text {th }}$ cause of failure. Here, $\omega_{j}(t)$ can be regarded as a prior guess at unknown hazard function specific to $j^{\text {th }}$ cause of failure while $c$ represents the degree of confidence in this guess.
The regression coefficients are assumed to be independently normal distributed, i.e.,

$$
\beta_{j} \sim N\left(\theta_{j}, \sigma_{j}^{2}\right), \quad j=1, \ldots, K,
$$

where $\beta_{j}, \theta_{j}$, and $\sigma_{j}^{2}$ are the regression coefficient, the mean, and the variance, respectively, specific to the $i^{\text {th }}$ cause of failure.

After defining the prior distributions, our interest turns to the joint posterior distribution:

$$
P\left(\beta, \Lambda_{0}, \mu \mid D\right) \propto L\left(D \mid \beta, \Lambda_{0}, \mu\right) \Pi(\beta) \Pi\left(\Lambda_{0}\right) \Pi(\mu),
$$

where $L$ denotes the likelihood function, $\Pi$ denotes the prior distribution, and $D$ denotes the observed data. Since (14) has a complicated form, we utilize the MCMC technique to generate random draws from the related full conditional posterior distributions, namely, $P\left(\beta \mid D, \Lambda_{0}, \mu\right), P\left(\Lambda_{0} \mid D, \beta\right.$, $\mu)$, and $P\left(\mu \mid D, \beta, \Lambda_{0}\right)$. These distributions need to be identified for the construction of an effective simulation method.

In order to assess the performance of our approach, we followed the cause-specific hazards-based simulation design of Beyersmann et al. [18]. All analyses were implemented in WinBUGS software [19] and $R$ statistical software version 2.6.2 [20].

\section{Results and Discussion}

3.1. Simulation Study. We considered a competing risks model with two causes of failure, where each has a Weibull distributed lifetime with parameters $\left(\lambda_{j}, \rho_{j}\right), j=1,2$, and set $\lambda_{1}=0.005, \lambda_{2}=0.003, \rho_{1}=1.9, \rho_{2}=1.3$. First, we simulate the failure times and the censored times. Then, we simulate the causes of failure and mask them randomly with equal chances to be masked or unmasked, which results in masked right-censored data. Last, we create inspection times so that the data becomes partly interval-censored data that includes exact, left-censored, right-censored, and intervalcensored failure times. The obtained data consists of $46 \%$ exact failure times, as well as 32\% right-, $10 \%$ left-, and $12 \%$ interval-censored failure times. Furthermore, $32 \%$ of the observations are masked while $30 \%$ and $6 \%$ of the observations fail due to causes 1 and 2, respectively.

A comparison of our approach on simulated partly interval-censored (PIC) data with the ones obtained using Sen's approach was done [2]. The reported results are based on five chains each of 4000 iterations with burn-in of 1000 iterations. We present the results from the simulated study in Table 1 and Figure 1. The results show that the estimates from the two approaches are comparable. Although our model deals with left-, right-, and interval-censored failure 


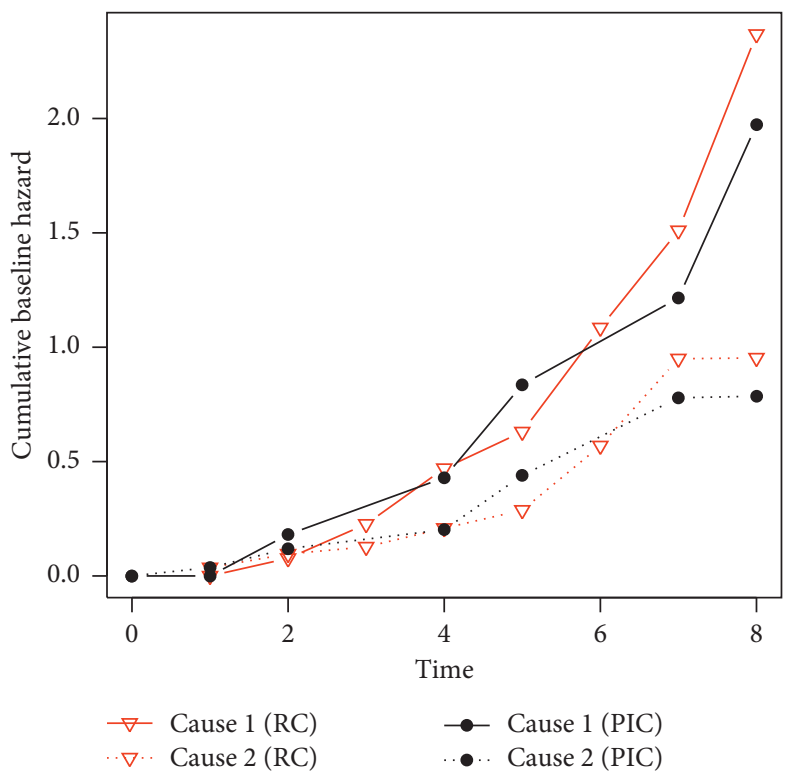

FIgURE 1: Comparison of cumulative baseline hazards from the two approaches, RC and PIC.

TABLE 2: Number of units across masking sets and failure/censored times.

\begin{tabular}{lcccccccc}
\hline Failure time type & & \multicolumn{3}{c}{ Masking sets } & & \\
& $\{0\}^{* *}$ & $\{\mathrm{~T}\}$ & $\{\mathrm{P}\}$ & $\{\mathrm{G}\}$ & $\{\mathrm{T}, \mathrm{P}\}$ & $\{\mathrm{T}, \mathrm{G}\}$ & $\{\mathrm{P}, \mathrm{G}\}$ & $\{\mathrm{T}, \mathrm{P}, \mathrm{G}\}^{*}$ \\
\hline Exact & 0 & 0 & 0 & 1 & 0 & 1 & 0 & 0 \\
Interval & - & 7 & 4 & 6 & 6 & 4 & 3 & 11 \\
Left & - & 3 & 0 & 0 & 1 & 1 & 1 & 4 \\
Right & 10 & - & - & - & - & - & - & 1 \\
Total & 10 & 10 & 4 & 7 & 7 & 6 & 4 & 1 \\
\hline
\end{tabular}

${ }^{*} \mathrm{~T}=$ turn; $\mathrm{P}=$ phase; $\mathrm{G}=$ ground. ${ }^{* *}\{0\}=$ no cause of failure or right-censored.

TABLE 3: The posterior summaries of the regression coefficients.

\begin{tabular}{lcccc}
\hline Parameters & Mean & Median & SE & $95 \%$ PCI \\
\hline$\beta_{0 \mathrm{~T}}$ & -21.49 & -21.32 & 6.744 & $(-35.33,-8.82)$ \\
$\beta_{0 \mathrm{P}}$ & -18.94 & -18.74 & 7.739 & $(-34.82,-4.36)$ \\
$\beta_{0 \mathrm{G}}$ & -20.96 & -20.83 & 7.424 & $(-36.11,-6.73)$ \\
$\beta_{1 \mathrm{~T}}$ & 28.67 & 28.39 & 13.71 & $(2.69,56.70)$ \\
$\beta_{1 \mathrm{P}}$ & 22.55 & 22.17 & 15.75 & $(-7.59,54.51)$ \\
$\beta_{1 \mathrm{G}}$ & 27.09 & 26.88 & 15.10 & $(-1.97,57.73)$ \\
\hline
\end{tabular}

times with considerable missing information, Table 1 shows that its posterior estimations of regression coefficients are reasonably close to those estimated by the model with only right-censored failure times. On the other hand, Figure 1 shows a comparison between the cumulative baseline hazards. The cumulative baseline hazards obtained from the two models are noticeably close with slight fluctuation for both causes (Figure 1).

3.2. Application to Electric Motors Data. We applied our approach to the data on failure times of insulation systems for electric motors (with their corresponding causes of failure), reported in Klein and Basu [21]. There are three common
TABle 4: Diagnostic probabilities of the full masked units.

\begin{tabular}{lccc}
\hline Diagnostic probability & \multicolumn{3}{c}{ Causes of failure } \\
& Turn & Phase & Ground \\
\hline$P^{3}$ & 0.289 & 0.444 & 0.267 \\
$P^{5}$ & 0.513 & 0.215 & 0.272 \\
$P^{11}$ & 0.498 & 0.245 & 0.257 \\
$P^{16}$ & 0.263 & 0.224 & 0.513 \\
$P^{17}$ & 0.290 & 0.204 & 0.506 \\
$P^{37}$ & 0.599 & 0.165 & 0.237 \\
$P^{38}$ & 0.353 & 0.189 & 0.459 \\
$P^{39}$ & 0.670 & 0.155 & 0.174 \\
$P^{40}$ & 0.614 & 0.167 & 0.219 \\
$P^{42}$ & 0.371 & 0.406 & 0.223 \\
$P^{54}$ & 0.375 & 0.402 & 0.223 \\
$P^{58}$ & 0.505 & 0.235 & 0.260 \\
\hline
\end{tabular}

types of insulation system failures: turn, phase, and ground. The experiment was conducted at three different stress levels $Z_{1}=(190+273.16) / 1000, Z_{2}=(220+273.16) / 1000$, and $Z_{3}=(240+273.16) / 1000$, where 20 units were tested at each level. To illustrate our approach, we converted this data into a PIC data with masked causes of failure. The obtained results are based on five chains where each is run 


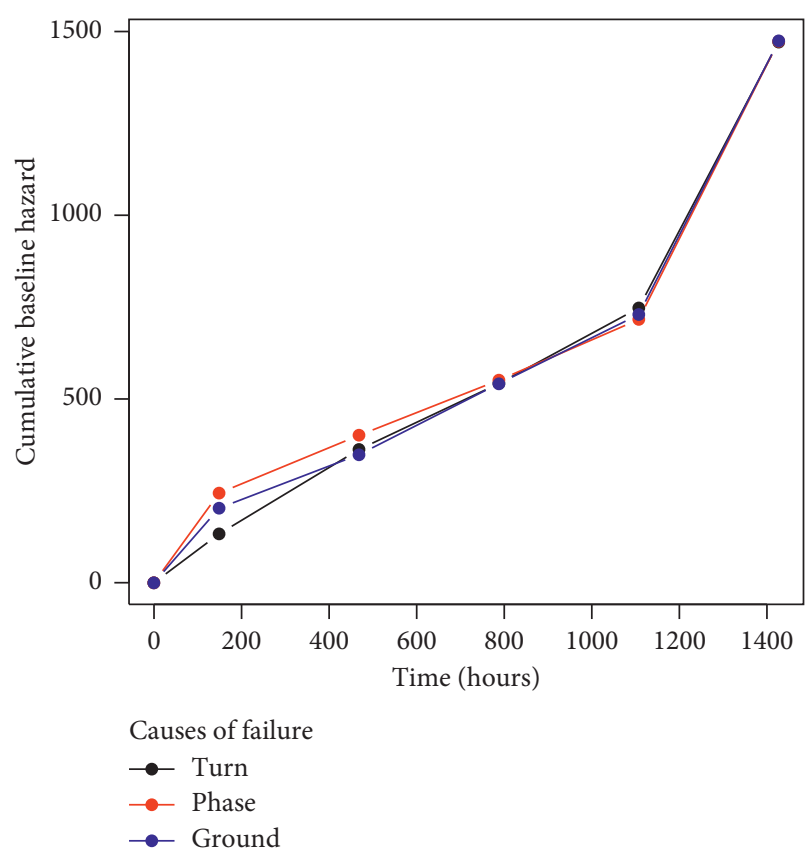

FIGURE 2: Cumulative baseline hazard of the three causes.

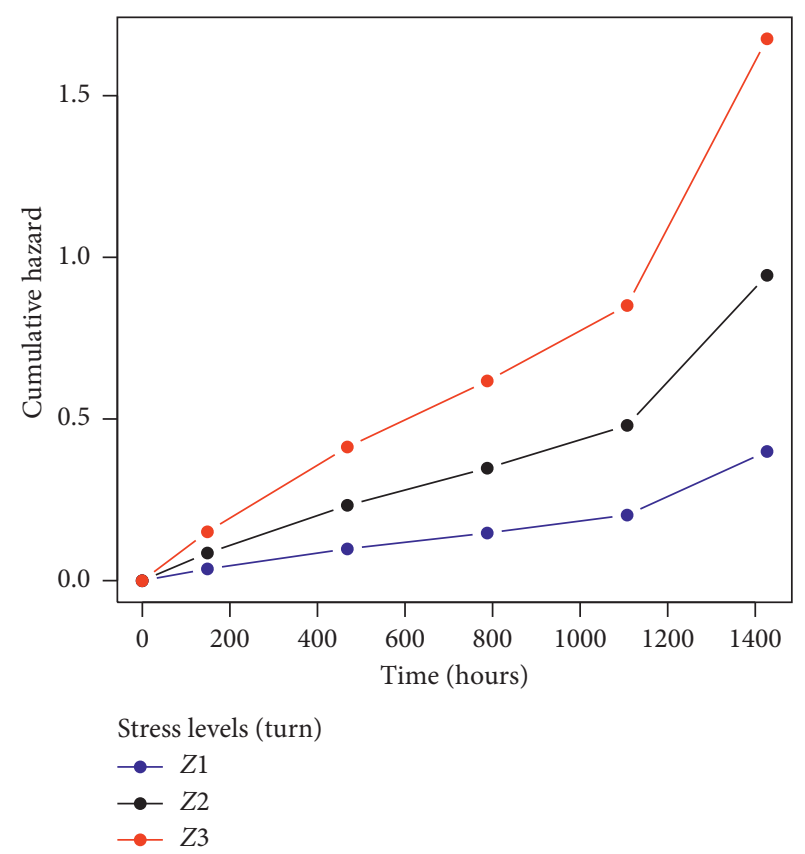

FIgURE 3: Cumulative hazard of cause turn.

with a burn-in of 20000 iterations, 50000 retained draws, and a thinning to every $15^{\text {th }}$ draw. Convergence is monitored and achieved for all parameters. Table 2 describes the number of units across the masking sets and the failure/censored times. Table 3 summarizes the posteriors estimates (mean, median, standard error (SE), and posterior credible interval (PCI)) of the regression coefficients while Table 4 shows the posterior mean of the diagnostic probabilities of the full masked units which are computed through equations (8) and (9). The results

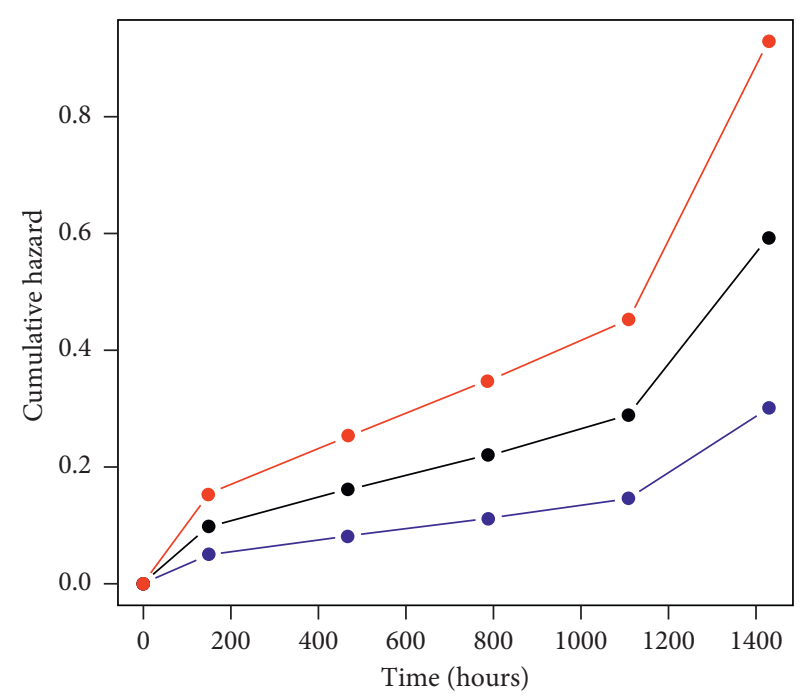

Stress levels (phase)

$\rightarrow Z 1$

$\rightarrow Z 2$

$\rightarrow Z 3$

FIgURE 4: Cumulative hazard of cause phase.

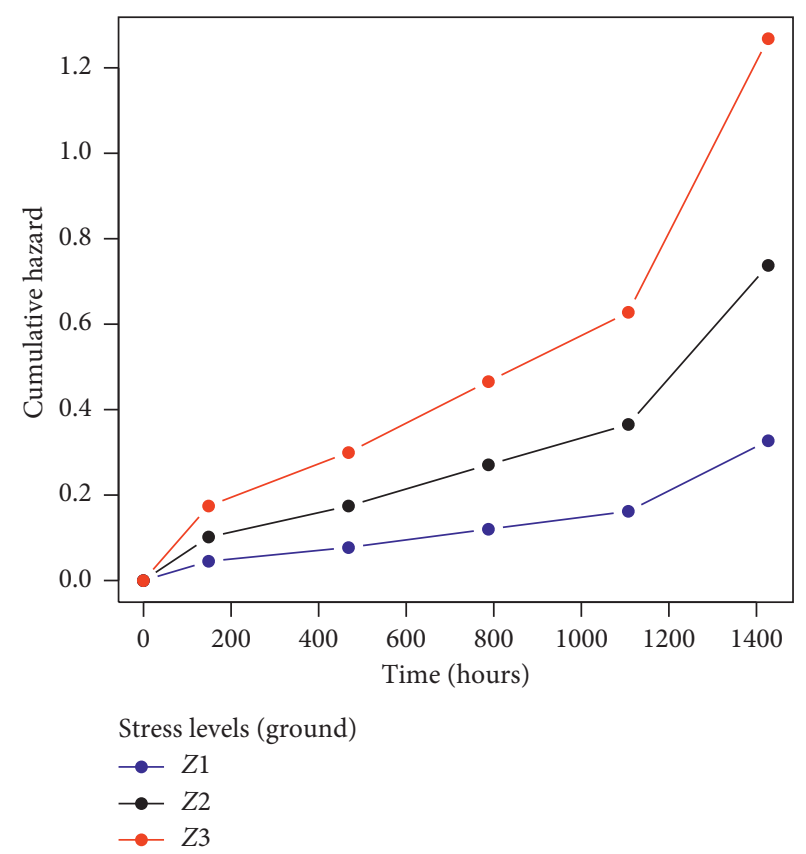

Figure 5: Cumulative hazard of cause ground.

indicate that $50 \%, 25 \%$, and $25 \%$ of the units fail due to turn, phase, and ground causes, respectively. Figure 2 depicts the cumulative baseline hazard functions of the three causes of failure. It is obvious from the figure that the three causes have almost the same cumulative hazard. In addition, Figures 3-5 demonstrate that the hazard increases with growing stress, irrespective of the cause. This is exactly the purpose of such experiments as it is run at high levels of stress to accelerate the failure as well as to reduce the cost and the experiment period. 


\section{Conclusion}

In this study, we have presented a Bayesian approach for the analysis of competing risks data with masked causes of failure. The Bayesian approach for competing risks models was derived where the cause of failure is masked and failure time is incomplete. This method offers some flexibility in modelling as it is not built on assumptions with questionable validity such as the symmetry assumption or independence of the competing risks. Furthermore, it provides an assessment of the risk factors' (covariates) effect on the hazard function. The results of the simulation support the feasibility of our modelling approach.

\section{Data Availability}

The data used to support the findings of this study are available within the article.

\section{Conflicts of Interest}

The authors declare that there are no conflicts of interest regarding the publication of this paper.

\section{Acknowledgments}

The authors would like to thank Mrs. Lynn Mason for editing the manuscript language. The publication of this article was funded by the Qatar National Library.

\section{References}

[1] B. Reiser, I. Guttman, D. K. J. Lin, F. M. Guess, and J. S. Usher, "Bayesian inference for masked system lifetime data," Applied Statistics, vol. 44, no. 1, pp. 79-90, 1995.

[2] A. Sen, M. Banerjee, Y. Li, and A.-M. Noone, "A Bayesian approach to competing risks analysis with masked cause of death," Statistics in Medicine, vol. 29, no. 16, pp. 1681-1695, 2010.

[3] C. Mukhopadhyay and S. Basu, "Bayesian analysis of masked series system lifetime data," Communications in StatisticsTheory and Methods, vol. 36, no. 2, pp. 329-348, 2007.

[4] C. Mukhopadhyay and A. P. Basu, "Bayesian analysis of incomplete time and cause of failure data," Journal of Statistical Planning and Inference, vol. 59, no. 1, pp. 79-100, 1997.

[5] S. Basu, A. Sen, and M. Banerjee, "Bayesian analysis of competing risks with partially masked cause of failure," Journal of the Royal Statistical Society: Series C (Applied Statistics), vol. 52, no. 1, pp. 77-93, 2003.

[6] S. Basu, A. P. Basu, and C. Mukhopadhyay, "Bayesian analysis for masked system failure data using non-identical Weibull models," Journal of Statistical Planning and Inference, vol. 78, no. 1-2, pp. 255-275, 1999.

[7] I. Guttman, D. K. J. Lin, B. Reiser, and J. S. Usher, "Dependent masking and system life data analysis: bayesian inference for two-component systems," Lifetime Data Analysis, vol. 1, no. 1, pp. 87-100, 1995.

[8] M. Miyakawa, "Analysis of incomplete data in competing risks model," IEEE Transactions on Reliability, vol. R-33, no. 4, pp. 293-296, 1984.

[9] G. E. Dinse, "Nonparametric prevalence and mortality estimators for animal experiments with incomplete cause-of- death data," Journal of the American Statistical Association, vol. 81, no. 394, pp. 328-336, 1986.

[10] L. Kuo and T. Y. Yang, "Bayesian reliability modeling for masked system lifetime data," Statistics \& Probability Letters, vol. 47, no. 3, pp. 229-241, 2000.

[11] A. Xu and Y. Tang, "Nonparametric bayesian analysis of competing risks problem with masked data," Communications in Statistics-Theory and Methods, vol. 40, no. 13, pp. 2326-2336, 2011.

[12] A. A. Neath and F. J. Samaniego, "On bayesian estimation of the multiple decrement function in the competing risks problem," Statistics \& Probability Letters, vol. 31, no. 2, pp. 75-83, 1996.

[13] B. J. Flehinger, B. Reiser, and E. Yashchin, "Parametric modeling for survival with competing risks and masked failure causes," Lifetime Data Analysis, vol. 8, no. 2, pp. 177-203, 2002.

[14] R. V. Craiu and B. Reiser, "Inference for the dependent competing risks model with masked causes of failure," Lifetime Data Analysis, vol. 12, no. 1, pp. 21-33, 2006.

[15] K. Lu and A. A. Tsiatis, "Multiple imputation methods for estimating regression coefficients in the competing risks model with missing cause of failure," Biometrics, vol. 57, no. 4, pp. 1191-1197, 2001.

[16] L. Han, Q. Gao, J. Yang et al., "Survival analysis of coal workers' pneumoconiosis (CWP) patients in a state-owned mine in the east of China from 1963 to 2014," International Journal of Environmental Research and Public Health, vol. 14, no. 5 , p. $489,2017$.

[17] S.-W. Liu, L.-C. Huang, W.-F. Chung et al., "Increased risk of stroke in patients of concussion: a nationwide cohort study," International Journal of Environmental Research and Public Health, vol. 14, no. 3, p. 230, 2017.

[18] J. Beyersmann, A. Latouche, A. Buchholz, and M. Schumacher, "Simulating competing risks data in survival analysis," Statistics in Medicine, vol. 28, no. 6, pp. 956-971, 2009.

[19] D. Spiegelhalter, A. Thomas, N. Best, and D. Lunn, WinBUGS User Manual Version 1.4, Kluwer Academic Publishers, Berlin, Germany, 2003.

[20] R Core Team, A Language and Environment for Statistical Computing, R. Foundation for Statistical Computing, Vienna, Austria, 2017.

[21] J. P. Klein and A. P. Basu, "Weibull accelerated life tests when there are competing causes of failure," Communications in Statistics-Theory and Methods, vol. 10, no. 20, pp. 2073-2100, 1981. 